\title{
Pyoderma Gangrenosum: A Review of Updates in Diagnosis, Pathophysiology and Management
}

\author{
Maria Skopis ${ }^{1, *}$ and Ayse Bag-Ozbek ${ }^{2}$ \\ 1 Department of Internal Medicine, Mount Sinai Medical Center, Miami Beach, FL 33140, USA \\ 2 Department of Internal Medicine, Division of Rheumatology, Allergy and Immunology, SUNY Stonybrook \\ University Hospital, Stonybrook, NY 11794, USA; abagozbek@gmail.com \\ * Correspondence: mskopis1115@gmail.com
}

Citation: Skopis, M.; Bag-Ozbek, A. Pyoderma Gangrenosum: A Review of Updates in Diagnosis,

Pathophysiology and Management. J 2021, 4, 367-375.

https://doi.org/10.3390/j4030028

Academic Editor: Maria

Luisa Balestrieri

Received: 6 May 2021

Accepted: 9 July 2021

Published: 19 July 2021

Publisher's Note: MDPI stays neutral with regard to jurisdictional claims in published maps and institutional affiliations.

Copyright: (C) 2021 by the authors. Licensee MDPI, Basel, Switzerland. This article is an open access article distributed under the terms and conditions of the Creative Commons Attribution (CC BY) license (https:/ / creativecommons.org/licenses/by/ $4.0 /)$.

\begin{abstract}
Pyoderma gangrenosum (PG) is a rare entity that is characterized by infiltration of neutrophils into the dermis, causing the formation of rapidly enlarging, painful and necrotic skin ulcers. The pathophysiology of PG is still poorly understood. However, genetic, autoimmune and autoinflammatory mechanisms have been proposed that could potentially explain the etiology of this ulcerating skin disorder. Early diagnosis and treatment are key, as the disease course is rapidly progressive and can leave disfiguring, cribriform scars. However, the diagnosis of PG proves difficult, firstly because there are multiple variants of the disease and secondly because it is a clinical diagnosis and can appear similar to that of other diseases such as vasculitis, skin/soft tissue infections and malignancy. Additionally, there are no official diagnostic criteria to aid in the recognition of PG, which often leads to significant delays in diagnosis. The treatment of PG consists in immunosuppression. However, due to a lack of standardized guidelines, therapeutic regimens are usually dependent upon the individual clinician's experience and are based on little evidence. Knowledge of the clinical features and pathophysiology of PG can aid in early diagnosis and targeted treatment strategies, which in turn results in improved patient outcomes.
\end{abstract}

Keywords: pyoderma gangrenosum; neutrophilic dermatosis; pathophysiology; pilosebaceous unit; $\mathrm{T}$ cell; neutrophil; autoinflammatory; autoimmune

\section{Methods}

The PubMed database was used to conduct an extensive literature search pertaining to the clinical manifestations, diagnosis, treatment and pathophysiology of pyoderma gangrenosum.

\section{Introduction}

Pyoderma gangrenosum was first described by the French physician Louis Brocq in 1908 when he observed a subset of patients who developed rapidly progressing necrotic skin ulcers with characteristically well- defined, blue, dusky, erythematous borders and purulent centers. It was initially thought that these lesions had an infectious origin, since hemolytic streptococci were cultured from the ulcers of patients with pyoderma gangrenosum [1]. Over time, however, it has been discovered that these lesions tend to occur in patients with concomitant systemic autoimmune illnesses such as inflammatory bowel disease, rheumatoid arthritis, systemic sclerosis and systemic lupus erythematosus, suggesting that there is a link between PG and autoimmunity [2,3]. Despite this observation, the exact mechanism of autoimmunity and the genetic mutations which predispose patients to develop these aberrant immune pathways remain poorly understood. In an effort to further understand this disease, the scientific community has researched the pathophysiology and genetics of PG, bringing forth interesting proposals throughout the years which could potentially explain the link between PG and autoimmunity. One of these theories suggests that PG and rheumatic diseases share a commonality in $\mathrm{T}$ cell abnormalities and 
that the same autoreactive $\mathrm{T}$ cells responsible for autoimmune disease also act to destroy pilosebaceous units in pyoderma gangrenosum. It has also been found that these effects are mitigated by the expression of certain genes (CXCL9, CXCL10, CXCL11) and via the secretion of certain interleukins (IL) (such as IL-8. IL-17, interferon gamma (IGFN), and IL-36G) by autoreactive T cells [4]. The most widely accepted theory is that PG is autoinflammatory in origin, driven by abnormalities of the innate immune system. There are several publications in support of this theory which will be discussed later in this review.

Early diagnosis of pyoderma gangrenosum is of essential importance, as it is key to initiating treatment and minimizing the occurrence of large, necrotic and extremely painful ulcers that can become secondarily infected and lead to the formation of disfiguring scars. The diagnosis of PG, however, can prove extremely difficult due to a lack of standardized clinical diagnostic criteria to aid in timely recognition and due to the fact that PG is a clinical diagnosis and the differential diagnosis for ulcerative lesions is quite vast. In fact, these lesions are often mistaken for cellulitis, necrotizing fasciitis, cryoglobulinemia and vasculitis, among several other conditions that can cause ulcerative lesions (for example, herpes simplex virus). Mistaking these lesions for necrotizing fasciitis is especially harmful, since, by the mechanism of pathergy, the debridement of PG lesions tends to exacerbate them [5]. Delays in diagnosis cause significant patient harm, as these ulcers progress rapidly leading to large, deep, extremely painful lesions which eventually become necrotic and lead to permanent disfigurement due to cribriform scar formation. Diagnostic criteria have been proposed to aid in the earlier recognition of PG, which will be discussed later in this review.

Due to the fact that the pathophysiology of PG is rooted in abnormalities of the immune system, it stands to reason that the cornerstone of treatment is immunosuppressive therapy. Regimens include intravenous or oral corticosteroids, cyclosporine, azathioprine, mycophenolate mofetil, methotrexate, colchicine, cyclophosphamide and tumor necrosis factor alpha inhibitors [5]. Other treatment options include intravenous immunoglobulins (IVIG), interleukin 12/23 inhibitors, IL-1 antagonists and IL-1 beta monoclonal antibodies [6]. Patients typically respond dramatically to immunomodulatory treatment with relief of pain and significant improvement in lesions rather quickly after the initiation of therapy. Prompt and appropriate treatment can stop new lesions from forming and help to slow the progression of existing lesions, thus preventing widespread cribriform scar formation. Due to the fact that the exact pathophysiology of this disease is not known, there are no standardized treatment guidelines or universally effective treatments for PG. Thus, therapeutic regimens are mostly anecdotal.

\section{Clinical Manifestations}

Pyoderma gangrenosum, along with Sweet syndrome and subcorneal pustular dermatosis, belongs to a subset of diseases called neutrophilic dermatoses. These diseases are inflammatory in nature and are characterized by a dense, mature neutrophilic infiltrate within the dermis or epidermis, which can eventually lead to suppurative folliculitis, leukocytoclastic vasculitis, fibrinoid necrosis and necrotizing granulomatous inflammation $[7,8]$. Pyoderma gangrenosum typically affects people between the ages of 20 and 50 years and is more commonly observed in women. These ulcerative lesions can occur anywhere on the body, but occur most commonly on the lower extremities [8]. Pyoderma gangrenosum lesions usually begin as small, tender papules which progress to large, necrotic and exquisitely painful ulcers. Patients with underlying comorbidities such as diabetes mellitus or peripheral vascular disease who develop PG display a slower, poorer wound healing.

The lesions of PG commonly occur in patients with underlying systemic illnesses such as inflammatory bowel disease (up to $30 \%$ ), rheumatoid arthritis and hematologic malignancies. The appearance of these lesions is often accompanied by systemic symptoms such as fever, malaise and arthralgias. In $25-50 \%$ of cases, lesions occur in places of previous minor injury by way of the pathergy phenomenon. Several subtypes of PG exist, including: classic, peristiomal, pustular, bullous and vegetative. However, classic ulcerative PG is the 
most common type. Classic PG occurs as a characteristic deep ulcer with jagged borders that has rapidly progressing zones of erythema and is exquisitely painful. These lesions eventually develop necrosis and form disfiguring cribriform scars. Peristomal PG occurs at sites where abdominal stomas have been constructed in patients post ileostomy or colostomy creation secondary to inflammatory bowel disease, malignancy or recurrent diverticulitis. Pustular PG is a rare form of the disease and is more superficial than the other variants. These lesions appear as small individual pustules that eventually fuse and ulcerate. Bullous PG is another superficial variant and occurs most commonly in patients with hematologic malignancies. The vegetative variant is also superficial and is less aggressive than the other types of pyoderma gangrenosum. Vegetative PG usually occurs as one solitary lesion and is more responsive to treatment than the other types [9].

\section{Pathophysiology}

The pathophysiology of PG has yet to be fully understood. Several hypotheses exist about the etiology of this ulcerating skin disease. Some studies suggest a complex interplay between genetics and autoimmunity as the underlying pathogenesis of pyoderma gangrenosum. Despite the fact that PG is classified as a neutrophilic dermatosis, one theory proposes that the pathophysiology of the disease is actually driven by autoreactive $\mathrm{T}$ cells which destroy pilosebaceous units. This is further evidenced by the fact that PG lesions do not typically occur in areas devoid of follicular adnexal structures such as the nipple-areolar complex, the palmar surface of the hand and the plantar surface of the foot. This theory proposes that a lack of pilosebaceous units in these areas confers a paucity of antigenic targets for T cells to destroy, and thus PG lesions do not form in these locations. The study also observed that PG scars showed a complete loss of pilosebaceous units after healing, meaning that PG ulcerations theoretically should not form in areas of previous scarring from other PG lesions. While this theory is undoubtedly interesting, it is important to note that the occurrence of a PG lesion on the plantar surface of the first metatarsophalangeal joint has been documented in the literature [10] in addition to the fact that there is a documented case of a large PG lesion occurring on the hypertrophic scar of a previous PG ulceration [11]. The formation of PG lesions in these areas, which lack pilosebaceous units, contradicts the theory that this is the main antigenic target of $\mathrm{T}$ cells during the formation of PG lesions. More research is needed to elucidate the exact antigenic targets involved in the formation of PG lesions, the exact role of $\mathrm{T}$ cells and the adaptive immune response in this inflammatory skin disease. Novel discoveries in these areas will be crucial to create new therapeutic targets for pyoderma gangrenosum.

In terms of the relationship between PG and autoimmune diseases, it is proposed that a commonality in $\mathrm{T}$ cell abnormalities and aberrant cytokine production are responsible for the increased incidence of PG in patients with diseases such as rheumatoid arthritis and inflammatory bowel disease $(2,4)$. One study found elevated levels of the cytokines CXC Motif Chemokine Ligand (CXCL) 9, CXCL10, CXCL11, interleukin (IL)-36G, IL-17A and interferon gamma in PG lesions. Neutrophil chemokines such as IL-8, chemokine C-C ligand-3 (CCL)-3 and CCL-5 were also detected in PG lesions, and the upregulation of transcription factors (STAT 1 and STAT 4) which promote Th1 differentiation was noted in patients with pyoderma gangrenosum (4). It has been shown that increased levels of IL-8 are implicated in PG, and one study found that injecting human skin grafts with IL-8 caused the formation of necrotic skin ulcers [12].

Currently, the most accepted theory regarding the pathophysiology of PG proposes that it is an autoinflammatory disease versus being autoimmune in origin. Autoinflammatory diseases are characterized by abnormalities in the innate immune response, whereas autoimmune diseases are characterized by aberrancies in the adaptive immune system. Unlike autoimmune disorders, autoinflammatory diseases do not have specific antigenic targets or high autoantibody titers [13]. Pyoderma gangrenosum is seen as part of the hereditary autoinflammatory syndromes PAPA (pyogenic arthritis, PG and acne), PASH (PG, acne and suppurative hidradenitis), SAPHO (synovitis, acne, pustulosis, hyperostosis, 
osteitis) and PAPASH (pyogenic arthritis, acne, PG and suppurative hidradenitis), which supports the theory that it is a disease rooted in an autoinflammatory origin. PAPA, PASH, PAPASH and SAPHO occur due to mutations in the genes responsible for activating the inflammasome, a group of proteins that are involved in initiating the innate immune response. It is believed that in the PAPA syndrome, the mutation of the proline-serinethreonine-phosphatase-interacting protein 1 (PSTPIP1) gene causes increased formation of inflammasomes via a heightened binding affinity to the protein pyrin (an inflammasome assemblage protein). These events eventually lead to the cleavage of inactive prointerleukin 1 beta to its active isoform interleukin 1 beta via the activation of the protease caspase 1 [14]. Elevated levels of interleukin- 1 in turn cause an increased endothelial cell expression of adhesion molecules and neutrophil recruitment [15].

Additionally, it has been reported that increased amounts of interleukin 1 beta and interleukin 1 beta receptors have been found in PG ulcers, which further implicates the role of interleukin-1 in the formation of PG lesions. This presents interleukin 1 beta as a therapeutic target for PG, and the literature reports a successful use of the interleukin $-1 \beta$ inhibitor canakinumab to treat these lesions [16]. A similar pathogenesis is implicated in the SAPHO syndrome, with genetic mutations in PSTPIP2 as the initiator of increased inflammasome formation and the aberrant activation of innate immunity. In PASH, genetic mutations known to occur in other autoinflammatory diseases such as PSTPIP1 (PAPA syndrome), MEFV (Familial Mediterranean Fever), NOD2 (Chron's Disease, Blau Syndrome) and NLRP3 (Muckle-Wells Syndrome) are implicated in its pathogenesis [14]. The overproduction of interleukin 1 beta also leads to the release of numerous pro inflammatory cytokines, including interleukin 17 (IL-17) and tumor necrosis factor alpha (TNF-alpha). Together, interleukin 1 beta, IL-17 and TNF-alpha cause a heightened production of matrix metalloproteinases, which cause tissue destruction [17].

Ortega-Loayza et al. defined the role of pattern recognition receptors (PRRs) (protein receptors which recognize characteristic antigenic patterns on pathogenic molecules and aid in the activation of the innate immune response) such as toll-like receptors (TLRs) in the pathogenesis of pyoderma gangrenosum. It was found that these receptors are upregulated in PG lesions, causing a higher rate of activation of the innate immune response in these areas. It should be noted that TLR overexpression has also been implicated in other autoimmune diseases such as rheumatoid arthritis and inflammatory bowel disease, implicating the role of these receptors in both autoimmune and autoinflammatory diseases. Interestingly, this study argues that IL- 1 beta is not specific to PG, as it is overexpressed in other conditions which may cause skin ulcerations. This notion is further supported by the fact that not all PG lesions respond to interleukin-1 inhibitors [18].

McKenzie et al. suggest that the pathophysiology of PG can actually be attributed to genetic mutations, causing a synergistic over activation of both the innate and adaptive immune systems via a trigger such as an external insult, like a minor trauma (aka pathergy). The exact mechanism by which this occurs, however, is poorly understood. This theory also emphasizes the role of interleukin 1 beta in the pathogenesis of PG as well as IL-12/23, IL-6 and a4b7 integrin [19]. Ortega-Loayza et al. found that genes which are implicated in the activation of both the innate and adaptive immune systems including STAT1, ILR1, +MAPK8, IRF3, NFKB1, MX1, HLA-A, TR4, TRL6, CD40, CD40LG, ITGAM and IRF7 were upregulated in PG lesions, supporting McKenzie et al.'s notion that both the adaptive and innate immune pathways play a role in the pathogenesis of pyoderma gangrenosum [18] Another interesting theory proposes that abnormal neutrophil metabolic fluctuations cause aberrancies in neutrophil activation, trafficking and signal transduction, leading to abnormal dermal neutrophilic infiltration and the formation of PG ulcers [20].

Despite the fact that much about the pathophysiology of PG is still unknown, the findings above indicate, overall, that PG and its associated syndromes can be classified as a spectrum of autoinflammatory diseases, all with the common pathophysiologic factor of genetic mutations which lead to increased inflammasome assembly and the upregulation of PRR's, causing an overactivation of the innate immune system. 


\section{Diagnosis}

The diagnosis of PG is difficult for several reasons. These lesions are similar in appearance to other neutrophilic dermatoses such as Sweet Syndrome and subcorneal pustular dermatosis and can also mimic necrotizing skin infections, vasculitis, arterial and venous insufficiency ulcers, cryoglobulinemia, hematologic issues such as Sickle cell disease, antiphospholipid antibody syndrome, calciphylaxis and even cutaneous Crohn's disease, making the differential diagnosis for PG quite large and resulting in significant delays in recognition and treatment [21]. A lack of standardized diagnostic and histological criteria adds an additional difficulty to recognizing PG lesions, thus making it a clinical diagnosis. Over the years, three diagnostic criteria have been proposed to aid in the recognition of pyoderma gangrenosum.

In 2004, Su et al. proposed guidelines consisting of two major and two minor criteria, also known as the Mayo diagnostic criteria, named after Dr. Su's work at the Mayo Clinic. The major criteria consist of: the presence of a necrotic, painful ulcer that is rapidly progressing with blue/violet, ragged borders and the exclusion of other diagnoses that could cause ulcerative skin lesions. The minor criteria consist of: rapid response to treatment with steroids (50\% improvement in lesions within 1 month), a history of pathergy, a history of systemic disease known to be associated with PG (inflammatory bowel disease, rheumatoid arthritis) and classic histologic findings of pyoderma gangrenosum. According to this tool, diagnosis of PG is made by fulfilling two major and two minor criteria [22].

14 years after Su et al. proposed their diagnostic criteria, Maverakis et al. presented additional diagnostic criteria based on an international Delphi consensus of experts that was found to yield a sensitivity of $86 \%$ and a specificity of $90 \%$ in diagnosing pyoderma gangrenosum if one major criterion and/or four minor criteria are met. The major criterion is the neutrophilic infiltration of the ulcer's edge. The minor criteria include: exclusion of soft tissue infection from the differential diagnosis, a history of pathergy, history of diseases associated with PG, like inflammatory bowel disease or rheumatoid arthritis, ulceration of a pustule, a papule or vesicle within 4 days of appearing, presence of an ulcerative lesion that has erythematous borders and is exquisitely tender, the presence of several ulcers, with at least one on the anterior surface of the lower extremity, the formation of scars that have a cribriform or "wrinkled paper" appearance and a decreased size of the ulcer within 1 month of starting immunosuppressive therapy [23].

In 2018, the PARACELSUS score was released by Dissemond et al. alongside Maverakis et al.'s international Delphi criteria as an additional set of proposed diagnostic criteria for pyoderma gangrenosum. This scoring system proposes 10 criteria, three of which are major and seven of which are minor. The three major criteria include: a rapidly progressive disease course, the thorough assessment and exclusion of alternate differential diagnoses and an erythematous-violet wound border. The seven minor diagnostic criteria include: alleviation of symptoms with immunosuppressive therapy, ragged borders of ulceration, excruciating pain described as $>4 / 10$ on the subjective visual analogue pain scale, lesions which occur exclusively at sites of trauma, histological evidence of suppurative inflammation, concomitant systemic disease and tissue erosion around the wound's borders. A score of greater than or equal to 10 out of 20 possible points affords a high probability that the lesion in question is PG and helps to differentiate PG ulcers from those of venous stasis. Dissemond et al. reported that the criteria had a sensitivity and specificity of $100 \%$ in diagnosing and differentiating PG lesions from venous stasis ulcers [24]. Given the high sensitivity and specificity of the PARACELSUS score in diagnosing PG, in addition to the fact that it is easily applicable and commonly used, we prefer to use this tool when diagnosing PG in clinical practice.

The efficacy of the Mayo diagnostic criteria, the international Delphi Criteria and the PARACELSUS diagnostic tools had not been compared until a cross- sectional retrospective cohort study in 2021 by Haag et al. examined the performance of the three diagnostic frameworks. The study found that the PARACELSUS criteria were the most successful diagnostic tool in identifying PG lesions (identified $89 \%$ of patients with PG) vs. the Mayo 
diagnostic criteria and the international Delphi criteria, which identified $74 \%$ of patients with pyoderma gangrenosum, respectively [25]. Future research is needed to refine the accuracy of these diagnostic tools, as all three frameworks include criteria which rely on the patient's history and physical examination-this information is subjective and dependent upon the individual interpretation of the clinician, which can vary amongst individuals and thus presents a challenge in providing a consistent, accurate diagnosis. Targeted objective markers are needed for a consistent and accurate diagnosis of PG, and hopefully, with continued research, the scientific community will be able to identify such markers.

In addition to a lack of standardized diagnostic criteria, there is also a lack of established, specific histologic criteria to diagnose pyoderma gangrenosum. Histology is nonspecific and differs with the age of the lesion. Usually, the main purpose of a skin biopsy is to exclude other diagnoses, and specimens can be sent for bacterial, fungal and mycobacterial cultures rather than for a definitive diagnosis. Classic ulcerative PG usually displays a sterile neutrophilic infiltrate in the dermis, abscess formation and neutrophilic pustules. Vasculitis is also sometimes seen on histology but is usually a result of the ulcerations themselves rather than a characteristic primary finding. If vasculitis is seen on histology, care must be taken to rule out true causes of both infection and vasculitis [21].

The above-proposed diagnostic criteria and histologic findings can aid in the prompt recognition of PG lesions, leading to early treatment and improved patient outcomes. Pyoderma gangrenosum remains an exclusively clinical diagnosis, however, and care must be taken to rule out necrotizing skin infection, malignancy and vasculitis before initiating immunosuppressive treatment. Due to these factors, the diagnosis remains challenging and requires a high index of suspicion after the thorough workup of the patient for other causes.

\section{Treatment}

A gold standard of therapy for PG does not exist, making the treatment of this skin condition extremely challenging. Due to a lack of randomized controlled trials exploring the most effective treatments for PG, most data on this subject come from case series and publications with poor evidence. Due to this paucity of data, the treatment of PG is largely based on the clinician's individual experience, and there are no solid therapeutic guidelines. Because we know that the pathophysiology of the disease is rooted in abnormalities of the immune system, it stands to reason that the cornerstone of treatment is immunosuppressive therapy. Therapeutic regimens differ with the severity and extent of the disease.

Initial steps in the treatment of PG include a proper wound care to prevent secondary bacterial infection, dressings, compression and adequate pain relief, as these lesions tend to be exquisitely tender and cause significant discomfort. Immunosuppressive therapy is given either topically or systemically, depending on the severity of the disease. Topical corticosteroids have been used to treat PG in addition to triamcinolone $40 \mathrm{mg} / \mathrm{mL}$ injected into the ulcer's edge (intralesional injections may worsen pathergy and, as such, must be used with caution). Topical tacrolimus has also been used to successfully treat lesions [9,21]. Severe disease requires treatment with systemic immunosuppressive therapy. In these instances, oral corticosteroids in a dose of $0.5-1 \mathrm{mg} / \mathrm{kg} /$ day are used, and pulsed doses of methylprednisolone $(1 \mathrm{~g} / \mathrm{d})$ for 5 days can also be given. A rapid regression of ulcers and pain relief has been observed with a high dose of corticosteroids, but they are not a long -term treatment strategy, as side effects include osteoporosis, adrenal suppression and increased risk of infection. Cyclosporine can be used to treat severe disease either alone or in combination with corticosteroids [20]. Other treatment options include azathioprine, dapsone, methotrexate, mycophenolate mofetil, sulfasalazine, colchicine, thalidomide and cyclophosphamide [5].

The anti- tumor necrosis factor alpha inhibitor infliximab has been shown to treat PG lesions effectively in addition to the other agents in this group, etanercept and adalimumab [26]. Anti- tumor necrosis alpha inhibitors were also found to facilitate a faster wound healing of PG lesions than steroids alone [27]. Additional therapeutic options used 
in the successful treatment of PG target other cytokines in the inflammatory cascade, including IL-12/IL-23 (ustekinumab) [28], IL-17 (secukinumab) [29], IL-1ß (canakinumab) [30], IL-1 Receptor I (anakinra) [31], IL-6 (tocilizumab) [19], JAK1,2 and 3 (tofacitinib and ruxolitinib) [32,33], and phosphodiesterase 4 (PDE4) (apremilast) [34]. There is also evidence that intravenous immune globulin (IVIG) can be used successfully as an adjuvant treatment for PG [35-37]. Clinical trials assessing the safety and efficacy of adalimumab in treating active PG lesions are recently underway, with one trial recently completed in Japan as of April 2020. Additionally, a clinical trial studying the safety and efficacy of JAK 1 inhibitor baricitinib will be underway in July 2021. The details of these clinical trials can be accessed by visiting the website clinicaltrials.gov, accessed on 19 July 2021. Additionally, it should be noted that of all the biologics studied, ustekinumab has proven a successful adjuvant treatment for all forms of PG, including severe refractory disease resistant to treatment with up to four other systemic treatments [38].

A particular therapeutic challenge is presented in patients with PG and hidradenitis suppurativa (HS) overlap syndrome. Cases of PG associated with HS are rare and often severe and refractory. Both of these skin diseases are autoinflammatory in origin and can also be seen together in the PASH and PAPASH syndromes. Treatment with tumor necrosis factor alpha inhibitors such as adalimumab and infliximab has proven effective in managing these challenging cases [39,40]. Additionally, interleukin 1-beta inhibitors have been described as an effective treatment for the HS/PG overlap syndrome, which stands to reason given the role of interleukin 1-beta in autoinflammatory diseases [41].

It should be noted that surgical debridement is usually contraindicated, as this tends to exacerbate PG ulcers via the mechanism of pathergy. There is, however, a population of patients with noninflammatory PG ulcers refractory to immunosuppressive treatment who have responded favorably to surgical intervention. One study looked at 126 cases of PG which were treated with operative management and found that only $16.7 \%$ had disease progression post-operatively [42]. Additionally, there is recent literature outlining the use of surgical therapy in conjunction with immunosuppression, negative pressure wound therapy and skin grafting to treat PG lesions. This method has proven to be a safe and effective treatment option, with one study showing the successful treatment of lesions in $86 \%$ of the patients observed [43]. Continued research and stronger evidence in the form of large randomized controlled trials is needed to determine new therapeutic targets and effective treatment strategies for pyoderma gangrenosum. Hopefully, with continued scientific discoveries regarding this subject, treatment will advance backed by reliable evidence, and the formation of solid treatment guidelines for PG will emerge in the coming years.

\section{Conclusions}

In conclusion, $\mathrm{PG}$ is a rare disease discovered over a hundred years ago and still largely shrouded in mystery; much about its pathophysiology, diagnosis and treatment remains unknown or difficult to determine. Scientific developments throughout the years have helped to unearth certain important aspects about the disease, including clues regarding its autoinflammatory origin, the cytokines involved in its pathogenesis that can serve as therapeutic targets and proposed diagnostic criteria by which we can recognize these lesions early on and initiate a prompt treatment. Due to the rarity of this condition, there is a paucity of solid evidence regarding the pathophysiology and treatment of pyoderma gangrenosum. The continued research on the subject provides reassurance that the scientific community is hard at work in developing an understanding of the underlying pathogenesis of PG, in an attempt to develop more targeted and efficient treatment strategies for patients suffering from these lesions. Early recognition coupled with tailored, effective and evidencebased treatment strategies will ensure a prompt diagnosis and a successful treatment of PG, which will ultimately result in an improvement in patient care and outcomes. 
Author Contributions: M.S., A.B.-O. made substantial contributions to the conception and design of this review and performed data interpretation. Both authors have read and agreed to the published version of the manuscript.

Funding: This research received no external funding.

Conflicts of Interest: The authors declare no conflict of interest.

\section{References}

1. Hobbs, M.M.; Ortega-Loayza, A.G. Pyoderma gangrenosum: From historical perspectives to emerging investigations. Int. Wound J. 2020, 17, 1255-1265. [CrossRef]

2. Skopis, M.; Elghawy, A.A.; Pociurko, B.; Oh, K.S.; Bag-Ozbek, A. Pyoderma gangrenosum associated with limited cutaneous systemic sclerosis: A rare case with literature review. Clin. Rheumatol. 2021, 40, 1141-1145. [CrossRef]

3. González-Moreno, J.; Ruíz-Ruigomez, M.; Rubio, J.L.C.; Fernández, R.R.; Centeno, N.O. Pyoderma gangrenosum and systemic lupus erythematosus: A report of five cases and review of the literature. Lupus 2014, 24, 130-137. [CrossRef] [PubMed]

4. Wang, E.A.; Steel, A.; Luxardi, G.; Mitra, A.; Patel, F.; Cheng, M.Y.; Wilken, R.; Kao, J.; De Ga, K.; Sultani, H.; et al. Classic ulcerative pyoderma gangrenosum is a T cell-mediated disease targeting follicular adnexal structures: A hypothesis based on molecular and clinicopathologic studies. Front. Immunol. 2018, 8, 1980. [CrossRef] [PubMed]

5. Goshtasby, P.; Chami, R.; Johnson, M. A novel approach to the management of pyoderma gangrenosum complicating reduc-tion mammaplasty. Aesthet. Surg. J. 2009, 30, 186-193. [CrossRef] [PubMed]

6. Quist, S.R.; Kraas, L. Treatment options for pyoderma gangrenosum. J. Dtsch. Dermatol. Ges. 2017, 15, 34-40. [CrossRef] [PubMed]

7. Cohen, P.R. Neutrophilic Dermatoses: A review of current treatment options. Am. J. Clin. Dermatol. 2009, 10, 301-312. [CrossRef]

8. Chakiri, R.; Baybay, H.; El Hatimi, A.; Gallouj, S.; Harmouch, T.; Mernissi, F.Z. Clinical and histological patterns and treatment of pyoderma gangrenosum. Pan Afr. Med. J. 2020, 36, 59. [CrossRef]

9. Brooklyn, T.; Dunnill, G.; Probert, C. Diagnosis and treatment of pyoderma gangrenosum. BMJ 2006, 333, 181-184. [CrossRef]

10. Saffie, M.G.; Shroff, A. A Case of pyoderma gangrenosum misdiagnosed as necrotizing infection: A potential diagnostic catastrophe. Case Rep. Infect. Dis. 2018, 2018, 1-5. [CrossRef]

11. Rout, A.N.; Sahu, K.; Sirka, C.S. Pyoderma gangrenosum over hypertrophic scar: Report of a rare presentation. Indian Dermatol. Online J. 2020, 11, 222-225. [PubMed]

12. Oka, M.; Berking, C.; Nesbit, M.; Satyamoorthy, K.; Schaider, H.; Murphy, G.; Ichihashi, M.; Sauter, E.; Herlyn, M. Interleukin-8 overexpression is present in pyoderma gangrenosum ulcers and leads to ulcer formation in human skin xenografts. Lab. Investig. 2000, 80, 595-604. [CrossRef]

13. Alberts, B.; Johnson, A.; Lewis, J.; Raff, M.; Roberts, K.; Walter, P. Molecular Biology of the Cell, 4th ed.; Garland Science: New York, NY, USA, 2002. Available online: https: / www.ncbi.nlm.nih.gov/books/NBK26846/ (accessed on 1 June 2021).

14. Marzano, A.; Borghi, A.; Meroni, P.L.; Cugno, M. Pyoderma gangrenosum and its syndromic forms: Evidence for a link with autoinflammation. Br. J. Dermatol. 2016, 175, 882-891. [CrossRef] [PubMed]

15. Galimberti, R.L.; Vacas, A.S.; Garlatti, M.L.B.; Torre, A.C. The role of interleukin- $1 \beta$ in pyoderma gangrenosum. JAAD Case Rep. 2016, 2, 366-368. [CrossRef] [PubMed]

16. Cugno, M.; Borghi, A.; Marzano, A.V. PAPA, PASH and PAPASH syndromes: Pathophysiology, presentation and treatment. Am. J. Clin. Dermatol. 2017, 18, 555-562. [CrossRef]

17. Rodríguez-Zúñiga, M.J.M.; Heath, M.S.; Gontijo, J.R.V.; Ortega-Loayza, A.G. Pyoderma gangrenosum: A review with special emphasis on Latin America literature. Bras. Dermatol. 2019, 94, 729-743. [CrossRef]

18. Ortega-Loayza, A.G.; Nugent, W.H.; Lucero, O.M.; Washington, S.L.; Nunley, J.R.; Walsh, S.W. Dysregulation of inflammatory gene expression in lesional and non lesional skin of patients with pyoderma gangrenousum. Br. J. Dermatol. 2018, 178. [CrossRef]

19. McKenzie, F.; Arthur, M.; Ortega-Loayza, A. Pyoderma gangrenosum: What do we know now? Curr. Dermatol. Rep. 2018, 7 , 147-157. [CrossRef]

20. Adachi, Y.; Kindzelskii, A.; Cookingham, G.; Shaya, S.; Moore, E.C.; Todd, R.F.; Petty, H.R. Aberrant neutrophil trafficking and metabolic oscillations in severe pyo-derma gangrenosum. J. Investig. Dermatol. 1998, 111, 259-268. [CrossRef]

21. George, C.; Deroide, F.; Rustin, M. Pyoderma gangrenosum-A guide to diagnosis and management. Clin. Med. 2019, 19, 224-228. [CrossRef]

22. Su, W.P.D.; Davis, M.D.P.; Weenig, R.H.; Powell, F.C.; Perry, H.O. Pyoderma gangrenosum: Clinicopathologic correlation and proposed diagnostic criteria. Int. J. Dermatol. 2004, 43, 790-800. [CrossRef] [PubMed]

23. Maverakis, E.; Ma, C.; Shinkai, K.; Fiorentino, D.; Callen, J.P.; Wollina, U.; Marzano, A.V.; Wallach, D.; Kim, K.; Schadt, C.; et al. Diagnostic criteria of ulcerative pyoderma gangrenosum: A delphi con-sensus of experts. JAMA Dermatol. 2018, 154, 461-466. [CrossRef]

24. Jockenhöfer, F.; Wollina, U.; Salva, K.; Benson, S.; Dissemond, J. The PARACELSUS score: A novel diagnostic tool for pyoderma gangrenosum. Br. J. Dermatol. 2019, 180, 615-620. [CrossRef] [PubMed]

25. Haag, C.; Hansen, T.; Hajar, T.; Latour, E.; Keller, J.; Shinkai, K.; Ortega-Loayza, A.G. Comparison of three diagnostic frameworks for pyoderma gangrenosum. J. Investig. Dermatol. 2021, 141, 59-63. [CrossRef] 
26. Reguiaï, Z.; Grange, F. The role of anti-tumor necrosis factor-?? Therapy in pyoderma gangrenosum associated with inflammatory bowel disease. Am. J. Clin. Dermatol. 2007, 8, 67-77. [CrossRef]

27. Argüelles-Arias, F.; Castro-Laria, L.; Lobatón, T.; Aguas-Peris, M.; Rojas-Feria, M.; Acosta, M.B.-D.; Soto-Escribano, P.; CalvoMoya, M.; Ginard-Vicens, D.; Chaparro, M.; et al. Characteristics and treatment of pyoderma gangrenosum in inflammatory bowel disease. Dig. Dis. Sci. 2013, 58, 2949-2954. [CrossRef] [PubMed]

28. A Vallerand, I.; Hardin, J. Ustekinumab for the treatment of recalcitrant pyoderma gangrenosum: A case report. SAGE Open Med. Case Rep. 2019, 7. [CrossRef] [PubMed]

29. McPHIE, M.L.; Kirchhof, M.G. Pyoderma gangrenosum treated with secukinumab: A case report. SAGE Open Med. Case Rep. 2020, 8. [CrossRef]

30. Koilos, A.G.; Maul, J.T.; Meier, B.; Traidl-Hoffmann, C.; Hertl, M.; Zillikens, D.; Röcken, M.; Ring, J.; Facchiano, A.; Mondino, C.; et al. Canakinumab in adults with steroid refractory pyoderma gangrenosum. Br. J. Dermatol. 2015, 173, 1216-1223. [CrossRef] [PubMed]

31. Brenner, M.; Ruzicka, T.; Plewig, G.; Thomas, P.; Herzer, P. Targeted treatment of pyoderma gangrenosum in PAPA (pyogenic arthritis, pyoderma gangrenosum and acne) syndrome with the recombinant human interleukin-1 receptor antagnoist anakinra. Br. J. Dermatol. 2009, 161, 1199-1201. [CrossRef]

32. Kochar, B.; Herfarth, N.; Mamie, C.; Navarini, A.A.; Scharl, M.; Herfarth, H.H. Tofacitinib for the treatment of pyoderma gangrenosum. Clin. Gastroenterol. Hepatol. 2019, 17, 991-993. [CrossRef]

33. Nasifoglu, S.; Heinrich, B.; Welzel, J. Successful therapy for pyoderma gangrenosum with a Janus kinase 2 inhibitor. Br. J. Dermatol. 2018, 179, 504-505. [CrossRef] [PubMed]

34. Laird, B.M.E.; Tong, L.X.; Sicco, K.I.L.; Kim, R.H.; Meehan, S.; Franks, J.A.G. Novel use of apremilast for adjunctive treatment of recalcitrant pyoderma gangrenosum. JAAD Case Rep. 2017, 3, 228-229. [CrossRef] [PubMed]

35. Song, H.; Lahood, N.; Mostaghimi, A. Intravenous immunoglobulin as adjunct therapy in refractory pyoderma gangrenosum: Systematic review of cases and case series. Br. J. Dermatol. 2018, 178, 363-368. [CrossRef]

36. Cummins, D.; Anhalt, G.; Monahan, T.; Meyerle, J. Treatment of pyoderma gangrenosum with intravenous immunoglobulin. Br. J. Dermatol. 2007, 157, 1235-1239. [CrossRef]

37. Walters, J.; Glover, S. IVIG treatment for refractory pyoderma gangrenosum in patients with inflammatory bowel disease. Am. J. Gastroenterol. 2012, 107, S503. [CrossRef]

38. Westerdahl, J.S.; Nusbaum, K.B.; Chung, C.G.; Kaffenberger, B.H.; Ortega-Loayza, A.G. Ustekinumab as adjuvant treatment for all pyoderma gangrenosum subtypes. J. Dermatol. Treat. 2021, 14, 1-5. [CrossRef] [PubMed]

39. Groleau, P.F.; Grossberg, A.L.; A Gaspari, A. Hidradenitis suppurativa and concomitant pyoderma gangrenosum treated with infliximab. Cutis 2015, 95, 26125210.

40. Reddick, C.L.; Singh, M.N.; Chalmers, R.J.G. Successful treatment of superficial pyoderma gangrenosum associated with hidrad-enitis suppurativa with adalimumab. Dermatol. Online J. 2010, 16, 15. [CrossRef] [PubMed]

41. Hsiao, J.L.; Antaya, R.J.; Berger, T.; Maurer, T.; Shinkai, K.; Leslie, K.S. Hidradenitis suppurativa and concomitant pyoderma gangrenosum. Arch. Dermatol. 2010, 146, 1265-1270. [CrossRef]

42. Haag, C.K.; Bacik, L.; Latour, E.; Morse, D.C.; Fett, N.M.; Ortega-Loayza, A.G. Perioperative management of pyoderma gangrenosum. J. Am. Acad. Dermatol. 2020, 83, 369-374. [CrossRef] [PubMed]

43. Eisendle, K.; Thuile, T.; Deluca, J.; Pichler, M. Surgical Treatment of Pyoderma Gangrenosum with Negative Pressure Wound Therapy and Skin Grafting, Including Xenografts: Personal Experience and Comprehensive Review on 161 Cases. Adv. Wound Care (New Rochelle) 2020, 9, 405-425. [CrossRef] [PubMed] 